\title{
Hidradenocarcinoma on the Orbit
}

\author{
Jaejoon Lim¹, Sang-Yeon Park², Helen Lew ${ }^{3}$, Kyunggi Cho ${ }^{1}$ \\ ${ }^{1}$ Department of Neurosurgery, CHA Bundang Medical Center, CHA University College of Medicine, Seongnam, \\ ${ }^{2}$ Dermay Dermatology Clinic, Hwaseong, ${ }^{3}$ Department of Ophthalmology, CHA Bundang Medical Center, CHA University \\ College of Medicine, Seongnam, Republic of Korea
}

\section{Corresponding author: Kyunggi Cho}

Department of Neurosurgery, CHA Bundang Medical Center, CHA University College of Medicine, 59, Yatap-ro, Bundang-gu, Seongnam 13496, Republic of Korea

Tel: +82-31-780-5688

Fax: +82-31-780-5269

E-mail: sandori50@gmail.com

Received: October 19, 2017

Revised: January 7, 2018

Accepted: January 18, 2018
Hidradenocarcinoma is a rare tumor of eccrine gland origin that has a predilection for the head and neck. It has an aggressive growth pattern and higher incidence of regional and distant metastases. Because hidradenocarcinoma shows very low incidence, the standard treatment is not yet established. We experienced an extremely rare case of hidradenocarcinoma with cancer associated retinopathy, so we report this case with literature review.

Key Words: Chemotherapy, adjuvant; Eccrine glands; Orbit; Radiotherapy, adjuvant; Sweat gland neoplasms

\section{INTRODUCTION}

Hidradenocarcinoma is a malignant tumor that originates from the eccrine gland, and it has a very aggressive pattern. Local invasion and distant metastasis occur frequently, and the recurrence rate after treatment is also high ${ }^{1,3,6)}$. So far, no standard treatment has been established because the incidence of hidradenocarcinoma is very low. The treatment option which most researchers have recommended is a complete resection ${ }^{2,13)}$. Adjuvant treatment including radiotherapy and chemotherapy also can be tried, but the effectiveness of these treatment regimens is not clear.

\section{CASE REPORT}

A 40-year old man presented with exophthalmus for 8 months and decreased vision for 1 month on left eye. The ophthalmological examination of left eye revealed visual field defect and decreased vision (0.2) and increased intraocular pressure (20 $\mathrm{mmHg}$ ). Computed tomography (CT) showed a well enhancing tumor in the left orbit with focal bone destruction of lateral orbital wall and focal invasion into the anterior portion of left cavernous sinus. Magnetic resonance image (MRI) revealed a localized mass at lateral aspect of left orbit measuring $4.5 \times \mathrm{x}$ $2.2 \times 3.6 \mathrm{~cm}^{3}$ in size which is iso-signal intensity on $\mathrm{T} 1$ and T2-weighted image, and heterogeneous enhancement at lateral aspect of left orbit. The focal bone destruction was seen at lateral orbital wall. The mass invaded into temporalis muscle, superior orbital fissure, and cavernous sinus (Fig. 1A-C). We performed a biopsy and the hidradenocarcinoma was diagnosed; hence, we planned to surgical resection with enucleation of eye ball. The grossly total resection was done through the modified lateral supraorbital approach (Fig. 2). The tumor appeared hyperemic and yellowish mass. Dissection the optic nerve
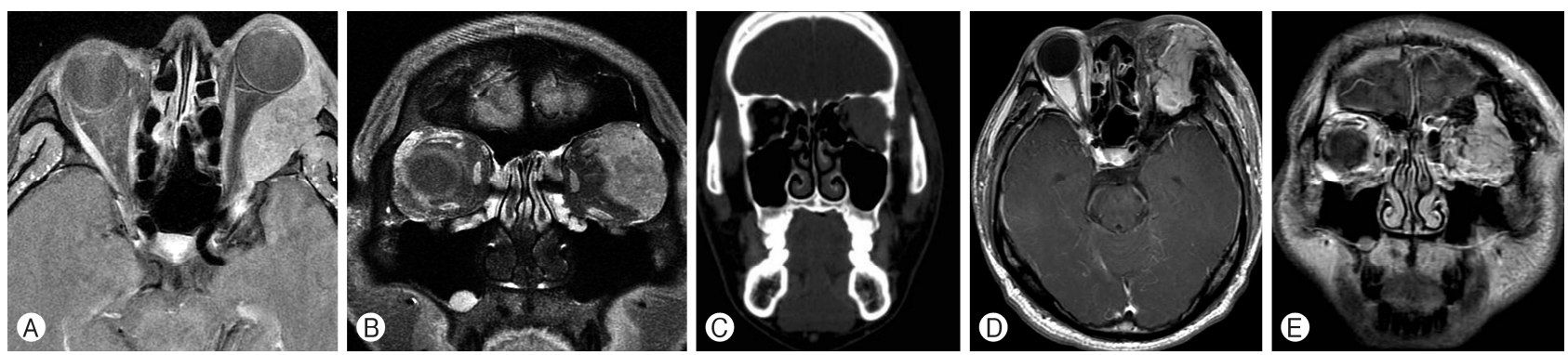

Fig. 1. Preoperative and postoperative magnetic resonance imaging (MRI) findings. (A-C) Preoperative MRI and computed tomography showed a heterogeneous enhanced mass with focal bony destruction. (D, E) The immediate postoperative MRI showed no residual tumor. 
from the tumor was difficult. The left eye ball was enucleated and fat packing was done. The immediate postoperative MRI showed no residual tumor.

In the biopsy of the tissue removed by surgery, ill-defined
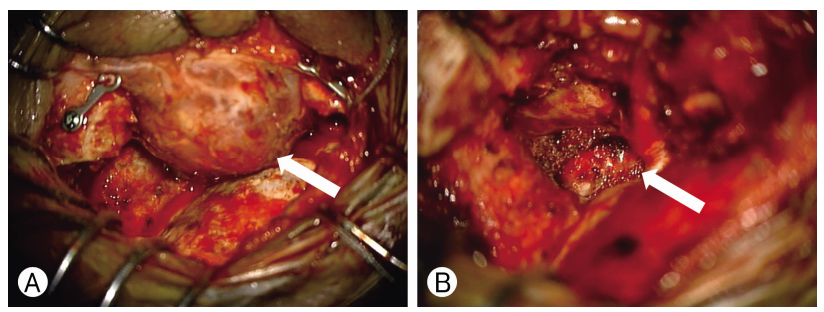

Fig. 2. The intraoperative tumor appearance. The patient underwent grossly total resection via the modified lateral supraorbital approach. (A) The tumor (arrow) appeared hyperemic and yellowish. (B) After the tumor was removed, the cavernous sinus (arrow) was seen.

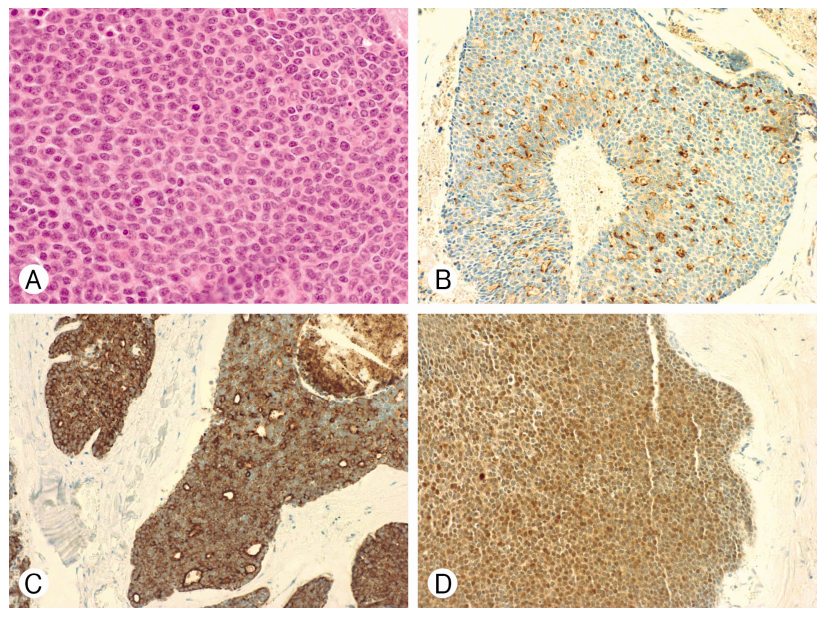

Fig. 3. Histopathological study of tumor. The ill-defined multilobular tumors are located in dermis. (A) There was significant nuclear atypia and moderate mitotic activity (hematoxylin and eosin stain [H \& E], $\times 200$ magnification). In immunohistochemical study, (B) epithelial membrane antigen, (C) cytokeratin 5/6, (D) S-100 were positive (B-D; $\times 100$ magnification).

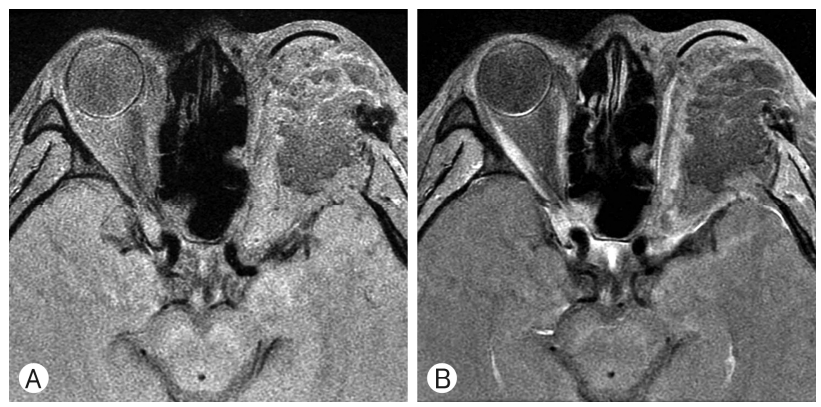

Fig. 4. Magnetic resonance imaging findings after 6 months of surgical resection. There was no evidence of recurrence. multilobular tumors were extensively observed in dermis. The tumors were surrounded by pseudocapsules consisting with fibrotic stroma, and a cystic cavity caused by tumor necrosis was also observed. The tumor cells were round to polygonal in shape with a clear cytoplasm. Cellular atypia and mitosis were observed frequently. Additionally, we performed immunohistochemical staining, and the tumors were positive for epithelial membrane antigen (EMA), vimentin, cytokeratin, S-100, and high proliferation labeling index (Ki-67 30\%), but they were negative for carcinoembryonic antigen (CEA) and gross cystic disease fluid protein 15 (GCDFP-15) (Fig. 3). Based on these histological findings and imaging study, we had diagnosed as hidradenocarcinoma on left upper eyelid extending into the left cavernous sinus with orbital bony destruction.

Considering the characteristics such as a very high recurrence rate and well metastasis of the tumor, adjuvant radiotherapy was performed with a total 5,400 cGy/27 fraction. The patient's status had been well maintained for 4 months without recurrence and complications.

The patient presented the sudden right eye visual symptom at 5 months after surgery. The ophthalmological examination of right eye revealed visual field defect and decreased vision (only light perception) and $16 \mathrm{mmHg}$ intraocular pressure. The mydriatic test result found a focal atrophy in the retina. In addition, the visual evoked potential result was that the P1 and P3 latency was delayed, and the electroretinography result found that the rod function was decreased. However, the multifocal electroretinogram was normal and both the MRI and positron emission tomography (PET)-CT image showed no recurrence and no metastasis (Fig. 4). We considered that the visual problem was associated with cancer associated retinopathy (CAR). And we started systemic steroid therapy and adjuvant chemotherapy. We administrated cisplatin and 5-fluorouracil. After the 1st cycle of chemotherapy, the patient's cognitive function was getting worse and other cranial nerve (III, IV, VI) palsy was developed. We recommended the MRI evaluation and 2nd cycle of chemotherapy, but the patient refused the additional therapy. The patient expired at 7 month later.

\section{DISCUSSION}

Hidradenocarcinoma was first described by Keasbey and Hadley $^{6}$ in 1954 . It is a very rare tumor, and Keasbey found only 3 cases of hidradenocarcinoma among the 235 sweat gland tumors when he first described this tumor in 1954. Mehregan et al. ${ }^{11)}$ mentioned that only 2 cases were diagnosed as hidradenocarcinoma among the 450,000 tissue samples collected for 20 years in the dermatopathology laboratory, and its prevalence is less than $0.001 \%{ }^{6,11)}$. So far, less than 70 cases have been reported. Patients with hidradenocarcinoma present with non-specific clinical symptoms. In most cases, a solitary cutaneous or subcutaneous lump for a long time period and the tumor size increases gradually. The tumor is often accom- 
panied by ulcers, and occasionally there are a variety of clinical manifestations such as fleshy color or red to gray color. It may occur anywhere including the head, torso, legs, and arms; but, it mainly occurs on the head and neck according to the reports. Also most of the tumor shows rapid growth over weeks to months, and it can cause aggressive local invasion and metastasis to the surrounding lymph nodes or other distant organs ${ }^{1,3,5,6,14,16)}$. In fact, hidradenocarcinoma has a poor prognosis, and the disease-free survival rate within 5 years is less than $30 \%$ according to the literature, and most of the reports suggested that distant metastasis is observed after tumor recurrence and eventually it leads to death ${ }^{8)}$. Statistically, the local recurrence rate after surgical resection is approximately $50 \%$, and more than $60 \%$ of the patients show metastasis to the lymph nodes, bones, skin, and internal organs ${ }^{13,15)}$.

Hidradenocarcinoma is a histopathologically ill-defined multilobular solid tumor located from the dermis to the subcutaneous tissue, and tumor necrosis is often observed in the lobule. The polygonal-shaped cells have oval nuclei and clear to slightly eosinophilic cytoplasm. The tumor cells show significant nuclear atypia and moderate mitotic activity ${ }^{1,3,11)}$. According to the literature, the immunohistochemical findings of hidradenocarcinoma are different, but most of the tumors are positive for vimentin, EMA, cytokeratin $5 / 6(\mathrm{CK} 5 / 6)$, and they show various responses to S-100, Ki-67, and GCDFP-15,7,12). In this case, the tumor was microscopically diagnosed as hidradenocarcinoma which was positive for vimentin, EMA, CK5/6, S-100, and high proliferation labeling index (Ki-67 30\%) and negative for GCDFP-15 and CEA.

No standard treatment has been established so far because hidradenocarcinoma has a very low incidence, and it is also challenging to treat the tumor due to its aggressive clinical characteristics. Wide resection is performed in most of the cases, and adjuvant therapy is considered as an additional treatment option. However, the prognosis of patients and clinical features still remain a matter of debate, ${ }^{9,10,146)}$. The wide local excision method may be a suitable local treatment option for non-disseminated lesions. However, although there is no evidence of distant metastasis and invasion of local lymph nodes on PET-CT, there is a possibility of microscopic invasion of lymph nodes in cases of locally aggressive invasion as in this case. Also, considering the high recurrence and metastatic rates of hidradenocarcinoma after surgery, we considered that combined treatment with wide excision and adjuvant radiotherapy would be appropriate for this patient. We performed adjuvant radiotherapy after complete surgical resection, and the patient is doing well and there has been no recurrence and complications for 4 months after surgery. However, 5 months after surgery, the patient's opposite eye showed symptoms of CAR. We administered chemotherapy, but 7 months later the patient was dead. CAR is a rare phenomenon associated with existing malignant tumor, and there is no standard treatment guideline. Appearance of CAR can imply that there can be a remaining malignant cells. In the case CAR occurring in the patient's opposite eye, we can consider that it would be a systemic metastasis.

We performed a gross total resection with postoperative radiotherapy and chemotherapy. And the patient was doing well for a relatively long time, considering the aggressive progression of hidradenocarcinoma. But, the disease progression was rapidly worsen after appearance of CAR. So, we considered that when hidradenocarcinoma is combined with cancer associated functional loss, it can act a poor prognosis factor.

\section{CONFLICTS OF INTEREST}

No potential conflict of interest relevant to this article was reported.

\section{REFERENCES}

1. Ahmed TSS, Priore JD, Seykora JT. Tumors of the epidermal appendages. In: Elder DE, Elenitsas R, Johnson BL, Murphy GF, Xu G eds. Lever's histopathology of the skin, ed 10. Philadelphia, PA: Lippincott Williams \& Wilkins, 2009, pp851-910

2. Arsalan-Werner A, Mentzel T, Kempf B, Sauerbier M: The hidradenocarcinoma of the wrist - an extremely rare malignant carcinoma: case presentation and literature review. Handchir Mikrochir Plast Chir 45:287-292, 2013

3. Brenn T, McKee PH. Tumors of the sweat glands. In: McKee PH, Calonje E, Granter SR eds. Pathology of the skin: With clinical correlations, ed 3. Philadelphia, PA: Elsevier Mosby, 2005, pp1589-1661

4. Farooq U, Choudhary S, Russo J, Vincek V, Elgart G: Clear cell hidradenocarcinoma with helpful immunohistochemistry: a case report. Int J Dermatol 52:1380-1382, 2013

5. Hernández-Pérez E, Cestoni-Parducci R: Nodular hidradenoma and hidradenocarcinoma. A 10-year review. J Am Acad Dermatol 12:15-20, 1985

6. Keasbey LE, Hadley GG: Clearcell hidradenoma; report of three cases with widespread metastases. Cancer 7:934-952, 1954

7. Ko CJ, Cochran AJ, Eng W, Binder SW: Hidradenocarcinoma: a histological and immunohistochemical study. J Cutan Pathol 33:726-730, 2006

8. Long WP, Dupin C, Levine EA: Recurrent malignant acrospiroma. Treatment by chest wall excision. Dermatol Surg 24:908912; discussion 911-902, 1998

9. Massad LS, Bitterman P, Clarke-Pearson DL: Metastatic clear cell eccrine hidradenocarcinoma of the vulva: survival after primary surgical resection. Gynecol Oncol 61:287-290, 1996

10. McCalmont TH: A call for logic in the classification of adnexal neoplasms. Am J Dermatopathol 18:103-109, 1996

11. Mehregan AH, Hashimoto K, Rahbari H: Eccrine adenocarcinoma. A clinicopathologic study of 35 cases. Arch Dermatol 119:104-114, 1983

12. Nazarian RM, Kapur P, Rakheja D, Piris A, Duncan LM, Mihm 
MC, Jr., et al.: Atypical and malignant hidradenomas: a histological and immunohistochemical study. Mod Pathol 22:600610, 2009

13. Ohta M, Hiramoto M, Fujii M, Togo T: Nodular hidradenocarcinoma on the scalp of a young woman: case report and review of literature. Dermatol Surg 30:1265-1268, 2004

14. Shu K, Xiao Q, Büchele F, Zhang S, Jiang W, Lei T: Diagnosis and treatment of clear cell hidradenocarcinoma of the scalp.
J Huazhong Univ Sci Technolog Med Sci 32:931-936, 2012

15. Wildemore JK, Lee JB, Humphreys TR: Mohs surgery for malignant eccrine neoplasms. Dermatol Surg 30:1574-1579, 2004

16. Yavel R, Hinshaw M, Rao V, Hartig GK, Harari PM, Stewart D, et al.: Hidradenomas and a hidradenocarcinoma of the scalp managed using Mohs micrographic surgery and a multidisciplinary approach: case reports and review of the literature. Dermatol Surg 35:273-281, 2009 